\title{
473879 - PALLIATION OF A TRACHEOBRONCHIAL ADENOID CYSTIC CARCINOMA USING A HARRELL Y STENT: THE COMPLEX ANESTHETIC CONSIDERATIONS OF A SHARED AIRWAY, A STENOTIC, NECROTIC TRACHEAL LESION AND BRONCHOSCOPIC STENT PLACEMENT
}

\author{
Carly Peterson, MD ${ }^{1}$, Hamed Umedaly, MD, FRCPC ${ }^{2}$ \\ 1. UBC Anesthesia Residency Program, UBC, Vancouver, BC, Canada \\ 2. VGH Department of Anesthesia, Vancouver Hospital and Health Science Center, \\ Vancouver, BC, Canada
}

Purpose: Inoperable tracheobronchial tumors carry a dismal prognosis and survival is mediated by complex palliative procedures that require a thorough understanding of the patient's disease and thoughtful communication between anesthesiologist and surgical team. This case allows us to present the multiple anesthetic considerations for a patient with a fixed stenotic tracheal lesion \& to illustrate the complexity of placing a Harrell Y Stent using both rigid and flexible bronchoscopy.

Clinical Features: The following case was compiled with the patient's consent. A 61 year old woman is admitted for her 400th GA for a planned tracheal stent replacement. In 1994 she was given the diagnosis of an inoperable adenoid cystic carcinoma encircling her mid-trachea for which she received radiation therapy. Hundreds of palliation procedures including Nd-YAG laser resections, tracheal toilets \& bronchoscopies to remove tumor debris as well as multiple stents of her trachea had given her a relatively good quality of life. She managed a complex medical regimen including multiple inhalers, chronic \& acute pain medications, anxiolytics, antitussives \& used portable HeliOx regularly. Pre-op she was dyspneic with severe inspiratory stridor but was known to be easy to ventilate \& to intubate. She was brought to the OR using her HeliOx \& premedicated with Glycopyrolate $0.4 \mathrm{mg}$, Midazolam 5mg \& Fentanyl 250mcg. Standard CAS monitors were applied. Remifentanil( $0.05 \mathrm{mcg} / \mathrm{kg} / \mathrm{min}) \&$ Propofol $(100 \mathrm{mcg} / \mathrm{kg} / \mathrm{min})$ infusions were combined with Sevoflurane. A Mivacurium $(12 \mathrm{mcg} / \mathrm{kg} / \mathrm{min})$ infusion was used for muscle relaxation. Manual ventilation was easy with good bilateral chest expansion. Rigid bronchoscopy was initiated \& Sanders jet ventilation was manually applied at 30psi, 15-25 bpm. The straight silicone stent was removed without concern. The patient was intubated with a size 8 ETT \& the flexible bronchoscope was passed through the lumen of the tube revealing necrotic tumor with purulent secretions obscuring much of the trachea. After vigorous suction \& ETT removal the rigid scope was reintroduced \& several attempts were made to place the Harrell Y-stent. Intermittent airway obstruction with dynamic pulmonary hyperinflation required suctioning of blood \& debris \& manual pressure on the sternum to assist with expiration. Difficulties deploying the stent were encountered. Sidestream capnometry was utilized via the air entrainment port of the rigid bronchoscope. This gave us an effective way to qualitatively monitor adequacy \& frequency of ventilation. When the waveform disappeared suddenly we were able to recognize airway obstruction before oxygen desaturation occurred. Eventually a size 9.0 ETT was placed just below the cords \& the stent was placed with forceps through the ETT. It was estimated that the ETTs, rigid \& flexible bronchoscope, 
stent \&/or forceps had crossed the cords approximately 30 times in 3 hours. Due to potential for laryngeal \& tracheal edema the patient was intubated \& ventilated for 6hrs post op. The patient was extubated fully awake \& breathing spontaneously in the PACU \& discharged home 3 days later.

Conclusion: Maintaining adequate oxygenation \& ventilation, ensuring excellent communication between the OR team \& the ability to adapt to a dynamic \& compromised airway were essential for effective anesthetic management of this complex patient.

References: 0 